\title{
Imbalanced Innate Lymphoid Cells are Associated With Disease Activity and Arthritis Involvement in Patients With Systemic Lupus Erythematosus
}

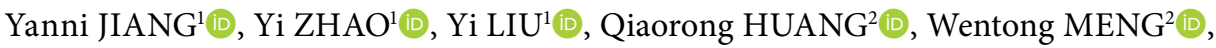 \\ Hong $\mathrm{XU}^{2} \mathbb{D}$, Xianming $\mathrm{MO}^{2} \mathbb{D}$ \\ ${ }^{1}$ Department of Rheumatology and Immunology, West China Hospital, Sichuan University, Chengdu, China \\ ${ }^{2}$ Laboratory of Stem Cell Biology, State Key Laboratory of Biotherapy, West China Hospital, Sichuan University and \\ Collaborative Innovation Center For Biotherapy, Chengdu, China
}

\begin{abstract}
Objectives: This study aims to evaluate the frequency and absolute number of circulating innate lymphoid cell (ILC) subsets and their associations with clinical and serological features in systemic lupus erythematosus (SLE).

Patients and methods: We recruited 28 SLE patients ( 6 males, 22 females; mean age 37.57 years; range, 18 to 56 years) and 13 healthy controls ( 4 males, 9 females; mean age 32.08 years; range, 19 to 48 years). Circulating ILC subsets were identified by flow cytometry. Associations between all detected cells and SLE disease activity, clinical manifestations, and serum autoantibodies were analyzed.

Results: In this study, significantly higher frequencies of ILC2s and ILC3s, lower frequencies of ILC1s, and higher ILC1/ILC3 and ILC1/ILC2 ratios were observed in SLE patients than in healthy controls. The frequencies and number of ILC3s were positively associated with SLE disease activity index 2000 score and anti-double stranded deoxyribonucleic acid titers in patients with SLE. Decreased ILC1 frequencies, increased ILC3 frequencies, and decreased ILC1/ILC3 and ILC2/ILC3 ratios were observed in patients with arthritis compared to those without arthritis.

Conclusion: Our results indicated biased altered distributions of circulating ILC subsets in SLE. ILC3s were associated with SLE disease activity, and ILC1s, ILC3s, and ILC1/ILC3 and ILC2/ILC3 ratios were associated with SLE accompanied with arthritis. Taken together, these results suggest that ILCs may serve as cellular biomarkers for disease activity and arthritis involvement in SLE.

Keywords: Arthritis, innate lymphoid cells, systemic lupus erythematosus.
\end{abstract}

Systemic lupus erythematosus (SLE) is a heterogeneous autoimmune disease characterized by the overproduction of autoantibodies and type I interferon (IFN). ${ }^{1}$ Previous studies have indicated that many immune cells, including B cells, T cells, natural killer (NK) cells, monocytes, and dendritic cells (DCs), participate in the pathogenesis of SLE, and abnormalities in these cells can occur at multiple points during the immune response, resulting in striking heterogeneity in clinical presentations. $^{2}$

Innate lymphoid cells (ILCs) represent a new lymphocyte subfamily that is distinct from $\mathrm{T}$ cells and $\mathrm{B}$ cells due to the absence of rearranged antigen-specific receptors, ${ }^{3}$ emerging as important effectors of innate immunity through their rapid production of proinflammatory and regulatory

\section{Received: April 08, 2019 Accepted: April 22, 2019 Published online: June 02, 2020}

Correspondence: Xianming Mo, MD. Department of Rheumatology and Immunology, West China Hospital, Sichuan University, Chengdu, China. Tel: 008602885164017 e-mail:xmingmoscu@163.com patients with systemic lupus erythematosus. Arch Rheumatol 2020;35(4):521-532. 
cytokines. ${ }^{4}$ Mimicking helper T (Th) cells, ILCs are divided into three subgroups based on the transcription factors they express and the cytokines they produce: group 1 ILCs (which include NK cells and ILC1s), group 2 ILCs (ILC2s), and group 3 ILCs (which consist of lymphoid tissue inducer and ILC3s). ${ }^{5}$ In addition, depending on the cytotoxic ability, ILCs are distributed into two groups as cytotoxic ILCs (NK cells) and non-cytotoxic or helper ILCs (also called CD127+ ${ }^{+}$ILCs, which are composed of ILC1s, ILC2s, and ILC3s). ${ }^{4}$

Accumulating evidences have shown that ILCs play an important role in the innate immune response against pathogenic microorganisms, tissue homeostasis, and metabolic regulation ${ }^{4}$ and in the development of chronic inflammatory and autoimmune diseases. ${ }^{6}$ Wohlfahrt et al. ${ }^{7}$ reported that the number of dermal and circulating ILC2s was increased in patients with systemic sclerosis and this increase was correlated with the extent of fibrosis. Rodríguez-Carrio et al. ${ }^{8}$ found increased numbers of ILC1s and ILC3s in lymph nodes of patients with rheumatoid arthritis (RA) compared to healthy controls. Recently, one report indicated that kidney-resident ILC2s were suppressed by IFN- $\gamma$ and that the progression of lupus nephritis was accompanied with a reduction of ILC2s in MRL/MpJ-Fas ${ }^{\mathrm{lpr}}$ mouse model. ${ }^{9}$ Cols et al. ${ }^{10}$ identified an expanded population of ILCs with a more remarkable IFN- $\gamma$ signature than $\mathrm{T}$ cells in both peripheral blood and gastrointestinal and lung tissues of patients with common variable immunodeficiency. Recently, two studies identified altered frequencies of circulating ILC1s, ILC2s, and ILC3s in patients with SLE compared to those in healthy controls. ${ }^{11,12}$

Thus, we hypothesize that an altered distribution of ILC subsets may play a role in the pathogenesis of human SLE based on the evidences about ILCs in autoimmune diseases and lupus animal models. In this study, we aimed to evaluate the frequency and absolute number of circulating ILC subsets and their associations with clinical and serological features in SLE.

\section{PATIENTS AND METHODS}

This cross-sectional, case-control study included 28 SLE patients (5 males, 23 females; mean age
37.57 years; range, 18 to 56 years) who fulfilled the American College of Rheumatology (ACR) classification criteria for SLE (1997) ${ }^{13}$ from the Department of Rheumatology and Immunology at West China Hospital, Sichuan University between April 2017 and December 2017. Patients that presented with other autoimmune diseases, infections, tumors or other severe conditions were excluded. We also recruited $13 \mathrm{sex}$ - and age-matched healthy controls (4 males, 9 females; mean age 32.08 years; range, 19 to 48 years). The study protocol was approved by the West China Hospital, Sichuan University Ethics Committee. A written informed consent was obtained from each participant. The study was conducted in accordance with the principles of the Declaration of Helsinki.Disease activity was measured using the SLE Disease Activity Index 2000 (SLEDAI-2000) and a score $\geq 5$ was used to define active SLE. ${ }^{14}$ Organ system involvement was identified according to the 1997 ACR criteria. Laboratory tests conducted included measuring titers of anti-nuclear antibody (ANA), anti-double stranded deoxyribonucleic acid (anti-dsDNA) antibody, anti-Sjögren's syndrome-related antigen A antibody, antiribonucleoprotein antibody, anti-ribosomal $\mathrm{P}$ antibody, and anti-Smith antibody, Coombs test, measuring serum levels of immunoglobulin (Ig)-G, IgA, IgM, complement component 3 (C3), C4, and C-reactive protein (CRP), and erythrocyte sedimentation rate (ESR) level. Disease manifestations and ongoing therapy were recorded at the inclusion visit.

Peripheral blood mononuclear cells (PBMCs) were separated from $4 \mathrm{~mL}$ of peripheral blood by Ficoll-Hypaque density-gradient centrifugation. Human Trustain FcX ${ }^{\mathrm{TM}}$ (BioLegend Inc., San Diego, CA, USA) was used to block the Fc receptor for 5 minutes prior to antibody staining. To eliminate nonspecific interactions between multiple BD Horizon Brilliant ${ }^{\mathrm{TM}}$ polymer conjugates, we used BD Horizon Brilliant Stain Buffer (BD Biosciences Inc., San Jose, CA, USA) according to the manufacturer's instructions. After adding all reagents, the cell suspension was incubated at room temperature in the dark for 15 minutes and analyzed using the CytoFLEX (Beckman Coulter Inc., S. Kraemer Boulevard, CA, USA) flow cytometer after washing twice with phosphate-buffered saline. The flow cytometry 
Table 1. Demographic, clinical, and serological features of systemic lupus erythematosus patients

\begin{tabular}{|c|c|c|c|c|c|c|c|}
\hline & \multicolumn{3}{|c|}{ SLE patients $(\mathrm{n}=28)$} & \multicolumn{3}{|c|}{ Healthy controls $(n=13)$} & \multirow[b]{2}{*}{$p$} \\
\hline & $\mathrm{n}$ & $\%$ & Mean \pm SD & $\mathrm{n}$ & $\%$ & Mean \pm SD & \\
\hline Age (year) & & & $37.6 \pm 2.3$ & 9 & 69.23 & $32.1 \pm 3.1$ & 0.169 \\
\hline $\begin{array}{l}\text { Sex } \\
\text { Female }\end{array}$ & 22 & 78.57 & & & & & 0.698 \\
\hline Disease duration (month) & & & $55.7 \pm 11.7$ & & & & \\
\hline Current concomitant medications & 27 & 96.43 & & & & & \\
\hline Hydroxychloroquine & 23 & 82.14 & & & & & \\
\hline Prednisone & 27 & 96.43 & & & & & \\
\hline Current dose of prednisone (mg/d) & & & $41.3 \pm 5.0$ & & & & \\
\hline Methotrexate & 14 & 50 & & & & & \\
\hline Cumulant dose of methotrexate & & & $1.0 \pm 0.3$ & & & & \\
\hline Other medications & 4 & 14.29 & & & & & \\
\hline SLEDAI-2000 & & & $8.9 \pm 0.8$ & & & & \\
\hline Active SLE patients (SLEDAI-2000 $\geq 5$ ) & 21 & 75 & & & & & \\
\hline Inactive SLE patients (SLEDAI-2000<5) & 7 & 25 & & & & & \\
\hline \multicolumn{8}{|l|}{ System involved (\% positive) } \\
\hline Mucocutaneous & 7 & 28 & & & & & \\
\hline Musculoskeletal & 6 & 21.43 & & & & & \\
\hline Neurological & 6 & 21.43 & & & & & \\
\hline Renal & 15 & 53.57 & & & & & \\
\hline Hematological & 11 & 39.29 & & & & & \\
\hline Mesenteric vasculitis (LMV) & 7 & 28 & & & & & \\
\hline \multicolumn{8}{|l|}{ Laboratory features (\% positive) } \\
\hline ANA & 28 & 100 & & & & & \\
\hline Anti-dsDNA antibody & 22 & 78.57 & & & & & \\
\hline Anti-Sm antibody & 8 & 28.57 & & & & & \\
\hline Anti-SSA antibody & 13 & 46.43 & & & & & \\
\hline Anti-RNP antibody & 15 & 53.57 & & & & & \\
\hline Anti-Rib antibody & 9 & 32.14 & & & & & \\
\hline Coombs test & 10 & 35.71 & & & & & \\
\hline $\mathrm{C} 3(\mathrm{~g} / \mathrm{L})$ & & & $0.6 \pm 0.0$ & & & & \\
\hline $\mathrm{C} 4$ (g/L) & & & $0.1 \pm 0.0$ & & & & \\
\hline $\operatorname{IgG}(\mathrm{g} / \mathrm{L})$ & & & $12.4 \pm 0.9$ & & & & \\
\hline $\mathrm{IgA}(\mathrm{mg} / \mathrm{L})$ & & & $2282.5 \pm 191.2$ & & & & \\
\hline $\operatorname{IgM}(\mathrm{mg} / \mathrm{L})$ & & & $965.5 \pm 102.3$ & & & & \\
\hline $\mathrm{CRP}(\mathrm{mg} / \mathrm{dL})$ & & & $6.1 \pm 1.7$ & & & & \\
\hline $\mathrm{ESR}(\mathrm{mm} / \mathrm{h})$ & & & $27.4 \pm 2.5$ & & & & \\
\hline
\end{tabular}

data were analyzed using the FlowJo X software (Treestar, Ashland, OR, USA). Gating was based on isotype controls and fluorescence minus one controls.

In humans, total ILCs are phenotypically identified as $\mathrm{CD}_{4} 5^{+}$PBMCs lacking markers specific for progenitor cells (CD34), $\mathrm{T}$ cells (CD3), B cells (CD19), NK cells (CD94 and CD16), DCs-monocytes (CD14, CD123, and $\mathrm{CD} 11 \mathrm{c})$, and mast cells (FceRI $\alpha$ ), i.e., lineage marker-negative (Lin) and CD127positive. ${ }^{15,16}$ ILC2s express surface marker 
(a)
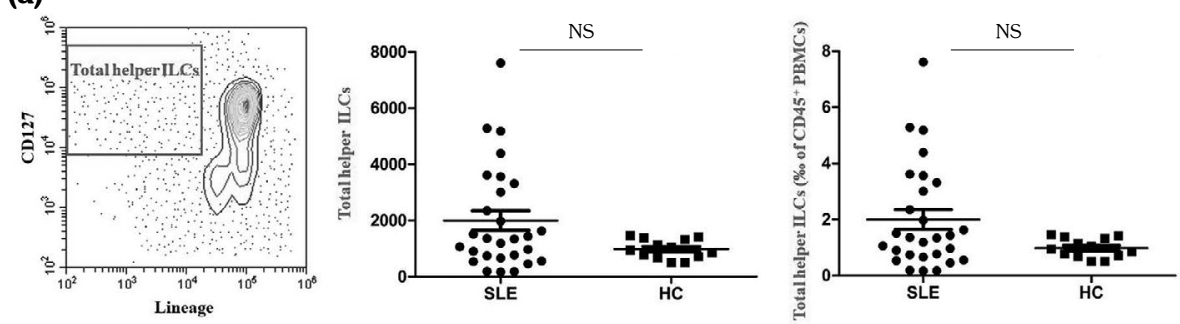

(b)
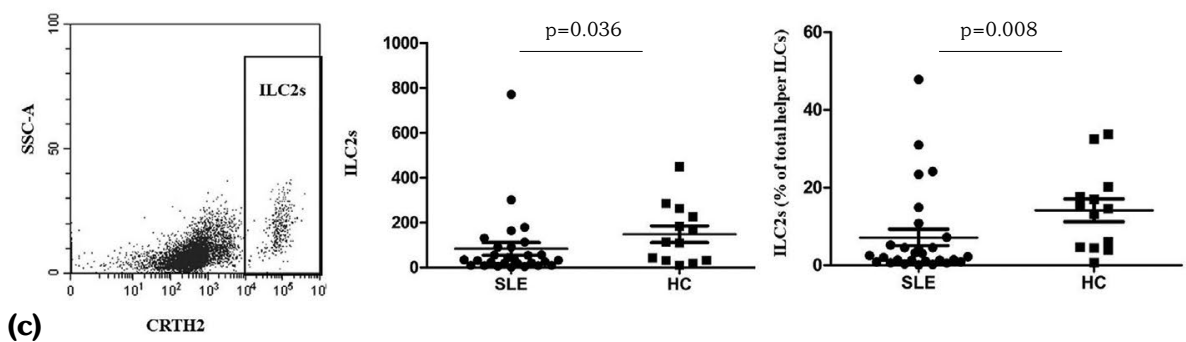

(c)
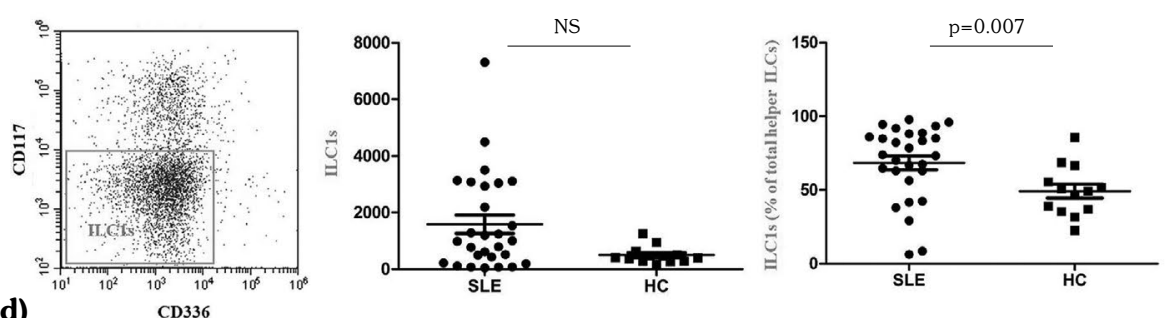

(d)
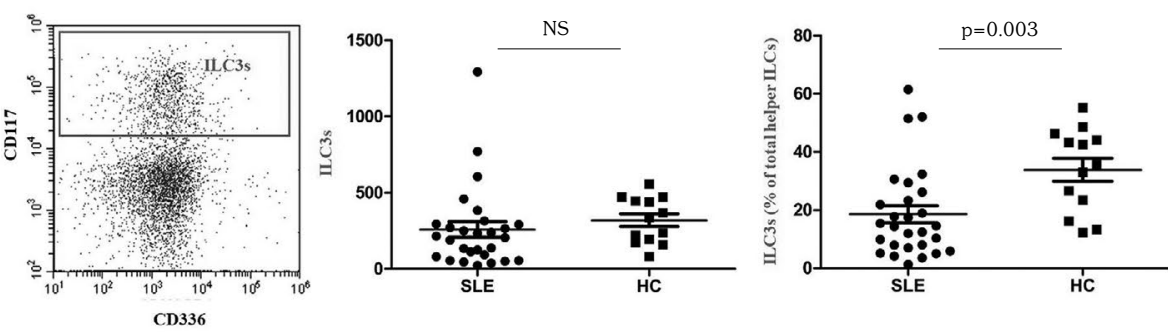

(e)
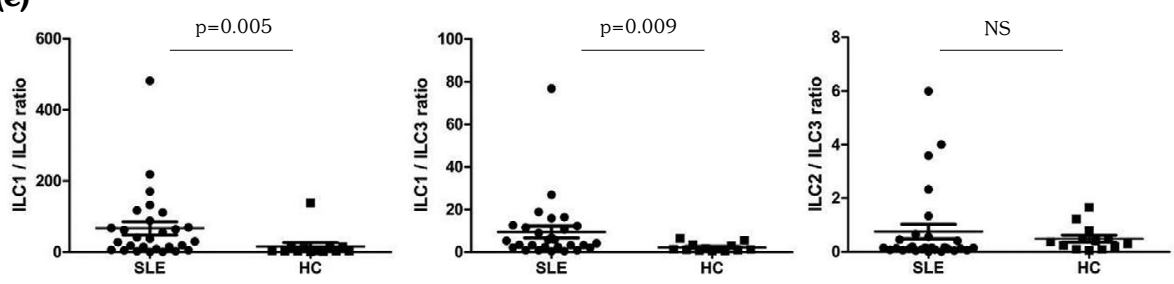

Figure 1. Differences in ILC subsets and ILC ratios between patients with SLE and HCs. Representative plots of ILCs and their subsets from SLE patients (left column). Plots are gated on live CD $45^{+}$PBMCs. Gates used to define populations under analysis are indicated: total helper ILCs (red gates, a), ILC1s (green gates, c), ILC2s (black gates, b), and ILC3s (blue gates, d). Absolute numbers (per $1 \times 10^{6} \mathrm{CD} 45^{+}$PBMCs; center column) and frequencies (for total helper ILCs based on CD45+ PBMCs and for three ILC subsets based on total helper ILCs, right column). Comparisons of total helper ILCs (a), ILC2s (b), ILC1s (c), and ILC3s (d); as well as ILC1/ILC2 ratio (e) (left column), ILC1/ILC3 ratio (center column), and ILC2/ILC3 ratio (right column) between SLE patients ( $\mathrm{n}=28$ ) and HCs $(n=13)$.

CD: Cluster of differentiation; NS: Not significant; SLE: Systemic lupus erythematosus; HC: Healthy control; SSC: Side scatter; ILC: Innate lymphoid cells; CRTH2: Chemoattractant receptor-homologous molecule expressed on TH2 cells; PBMC: Peripheral blood mononuclear cells; Data are shown as mean \pm standard error of mean. Differences were assessed by Mann-Whitney U test. $\mathrm{P}<0.05$ were considered statistically significant. 
chemoattractant receptor-homologous molecules expressed on Th2 cells (CRTH2), whereas ILC3s do not express CRTH2 but express CD117. ILC1s lack CRTH2, CD117, and CD336 expression, allowing us to distinguish these three ILC subsets based on CRTH2, CD117, and CD336 expression. The eight-color immunophenotyping panel is described in Table 1. The gating strategy and definition of all immune cells are shown in Figure 1 and Table 2, respectively.

\section{Statistical analysis}

Statistical analyses were performed using IBM SPSS version 22.0 software (IBM Corp., Armonk, NY, USA) and Prism 5.0 (GraphPad Software, Inc., La Jolla, CA, USA). Continuous variables were expressed as the mean \pm standard error of the mean, and categorical variables were reported as numbers (\%). Differences between two groups were compared using the MannWhitney U test and Fisher's exact test for categorical variables. Differences between the three study groups were analyzed using KruskalWallis followed by a Dunn's test. Correlation analysis was evaluated using the Spearman correlation coefficient. $\mathrm{P}<0.05$ was considered statistically significant.

\section{RESULTS}

In the present study, all enrolled patients $(n=28)$ were Han Chinese with a mean age of 37.57 years, and $82.14 \%$ of the patients (23) were females. Patients with a mean SLEDAI-2000 score of 8.93 were enrolled. The mean disease duration was 55.68 months. The other clinical manifestations and serological features of the recruited patients are summarized in Table 1.

First, we observed no significant differences in the absolute numbers of total ILCs, ILC1s, and ILC3s and frequencies of total ILCs in patients with SLE compared to healthy controls. However, absolute numbers of ILC2s were significantly decreased in patients with SLE compared to those in healthy controls (normalized per $1 \times 10^{6}$ total CD45+ PBMCs; 83.0 \pm 28.5 and $148.2 \pm 36.5$, respectively; $p=0.036)$ (Figure 1b). Decreased frequencies of ILC2s $(7.2 \pm 2.1 \%$ and $14.1 \pm 2.9 \%$, respectively; $p=0.008$ ) (Figure $1 \mathrm{~b}$ ) and ILC3s $(18.6 \pm 2.9 \%$ and $33.9 \pm 4.0 \%$, respectively; $p=0.003$ ) (Figure 1d) were observed in SLE patients compared with healthy controls. In contrast, frequencies of ILC1s were higher in SLE patients than in healthy controls $(68.4 \pm 4.5 \%$ and $49.3 \pm 4.8 \%$, respectively; $p=0.007$ ) (Figure 1c).

Next, we analyzed the ratios of ILC1s to ILC2s (ILC1/ILC2), ILC1s to ILC3s (ILC1/ILC3), and ILC2s to ILC3s (ILC2/ILC3) in SLE patients and healthy controls. A significantly higher ILC1/ILC2 ratio $(66.6 \pm 18.6$ and $15.8 \pm 10.3$, respectively; $\mathrm{p}=0.005)$ and a higher ILC1/ILC3 ratio $(9.4 \pm 2.8$ and $2.1 \pm 0.5$, respectively; $p=0.009$ ) were observed in patients with SLE than in healthy controls. However, there was no difference in the ILC2/ILC3 ratio between the SLE patients and healthy controls (Figure 1e).

Furthermore, we analyzed the potential associations of ILC subsets and their ratios with SLE disease activity. We grouped patients with SLE into patients with inactive SLE $(n=7)$ and patients with active SLE $(\mathrm{n}=21)$ according to the SLEDAI-2000 score. We compared the differences of cell population numbers, frequencies, and ratios among healthy controls, patients with active SLE, and patients with inactive SLE. We found significantly increased frequencies of ILC1s in patients with active SLE compared to those in healthy controls $(68.2 \pm 6.0 \%$ and $49.3 \pm 4.8 \%$, respectively; $p=0.028$ ) (Figure $2 \mathrm{~d}$ ), decreased frequencies of ILC2s in patients with active SLE compared with those in healthy controls $(3.8 \pm 1.2 \%$ and $14.1 \pm 2.9 \%$, respectively; $\mathrm{p}=0.008$ ) (Figure 2f), and decreased frequencies of ILC3s in patients with inactive SLE compared with those in healthy controls $(10.7 \pm 2.3 \%$ and $33.9 \pm 4.0 \%$, respectively; $\mathrm{p}=0.005$ ) (Figure $2 \mathrm{~h}$ ). In addition, decreased absolute numbers of ILC3s in patients with inactive SLE compared with healthy controls $(105.9 \pm 23.8$ and $319.4 \pm 41.8$, respectively; $\mathrm{p}=0.006$ ) (Figure $2 \mathrm{~g}$ ) and increased absolute numbers of ILC3s in patients with active SLE compared to patients with inactive SLE $(309.3 \pm 63.4$ and $105.9 \pm 23.8$, respectively; $\mathrm{p}=0.011$ ) (Figure $2 \mathrm{~g}$ ) were observed in the present study. Moreover, a higher ILC1/ILC2 ratio in patients with active SLE compared with that in healthy controls $(73.1 \pm 23.6$ and $15.8 \pm 10.3$, respectively; $p=0.009$ ) (Figure $2 \mathrm{i}$ ) and a higher ILC1/ILC3 ratio in patients with inactive SLE than in healthy controls $(9.3 \pm 2.5$ and $2.1 \pm 0.5$, respectively; $\mathrm{p}=0.012$ ) (Figure $2 \mathrm{j}$ ) were also 
(a)

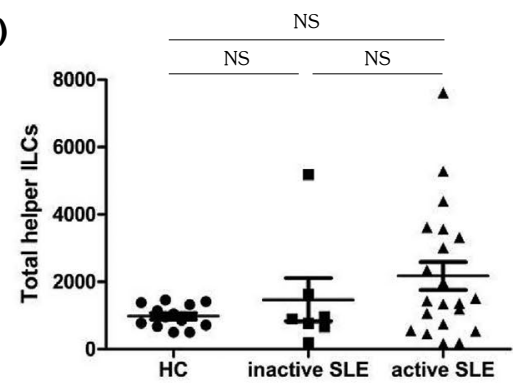

(c)

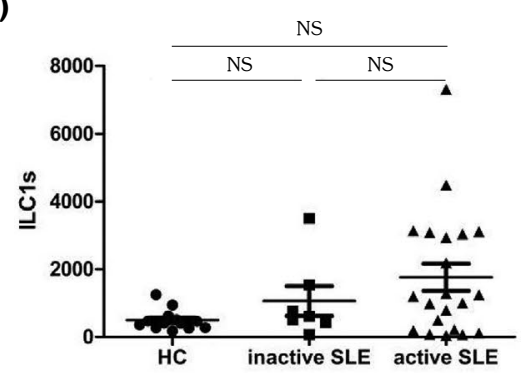

(e)

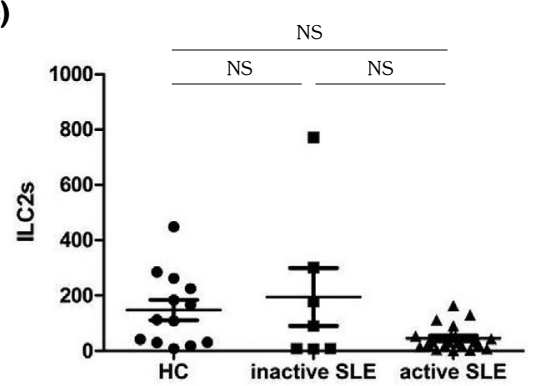

(g)

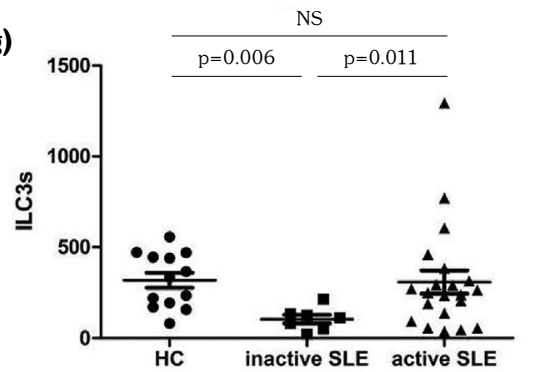

(b)

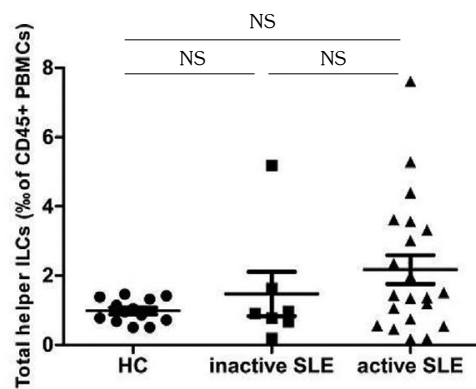

(d)

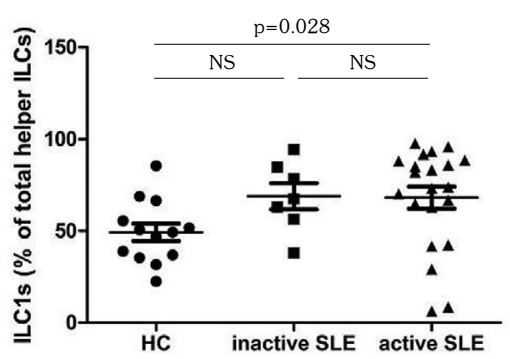

(f)

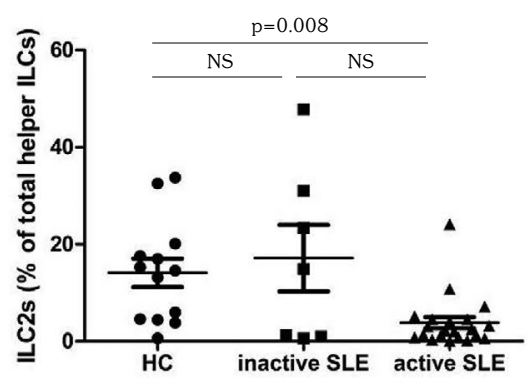

(h)

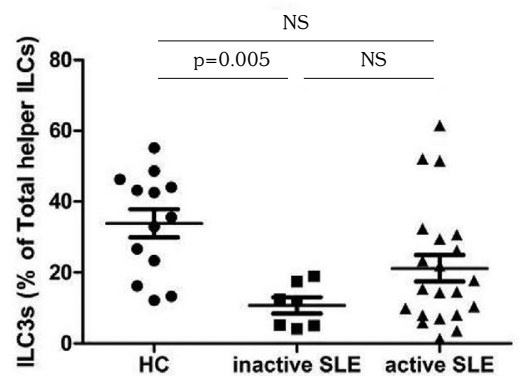

(k) (i)

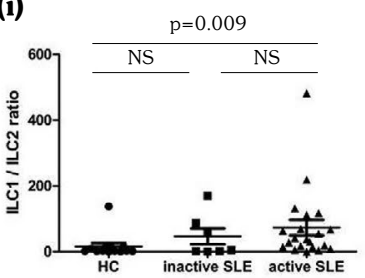

(j)

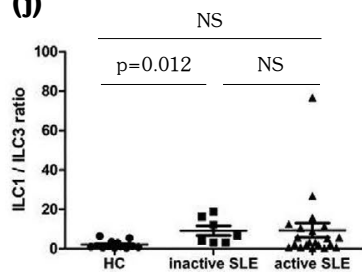

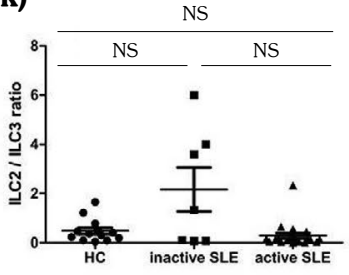

Figure 2. Differences in ILC subsets and ILC ratios among patients with active SLE, inactive SLE, and HCs. Comparisons of absolute numbers and frequencies of total helper ILCs (a and b), ILC1s (c and d), ILC2s (e and f), ILC3s (g and h), ILC1/ILC2 ratio (i), ILC1/ILC3 ratio (j), and ILC2/ILC3 ratio (k) among patients with active SLE.

Systemic Lupus Erythematosus Disease Activity Index 2000 (SLEDAI-2000) $\geq 5, n=21$ ], patients with inactive SLE (SLEDAI-2000 <5, $n=7$ ), and HCs ( $n=13$ ); ILC: Innate lymphoid cell; NS: Not significant; HC: Healthy control; SLE: Systemic lupus erythematosus; PBMC: Peripheral blood mononuclear cell; Data are shown as mean \pm standard error of mean. Differences were assessed by Kruskal-Wallis followed by Dunn's test. $\mathrm{P}<0.05$ were considered statistically significant. 
(a)

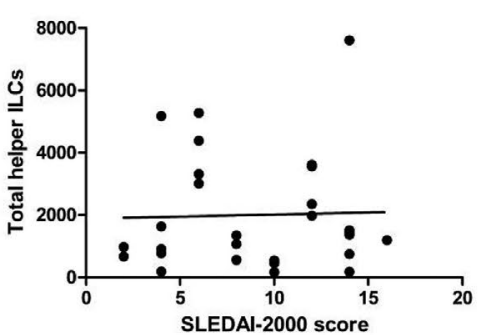

(c)

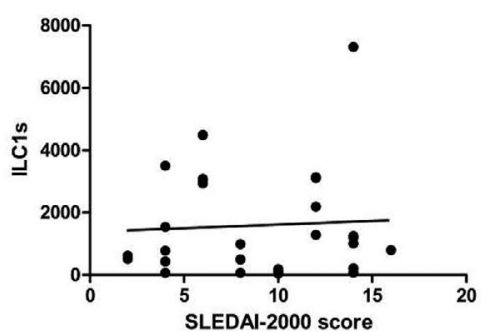

(e)

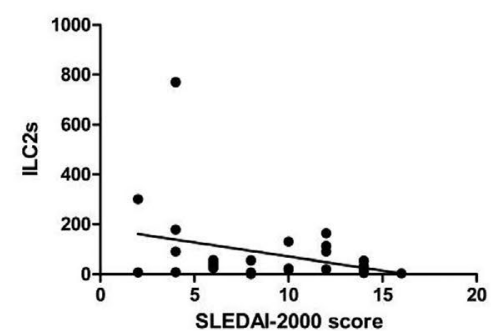

(g)

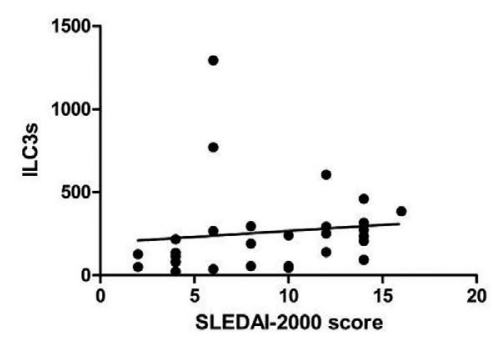

(b)

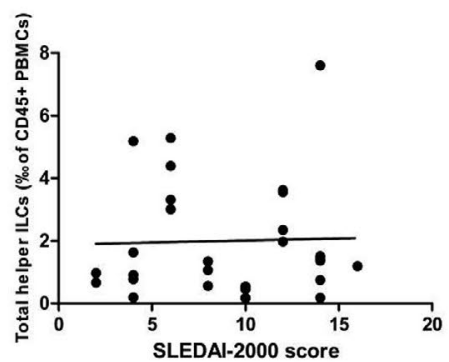

(d)

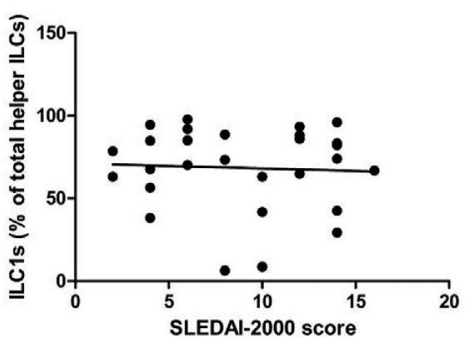

(f)

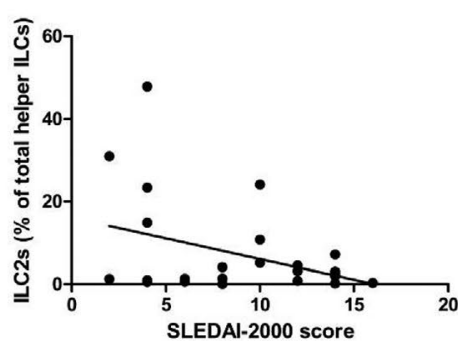

(h)

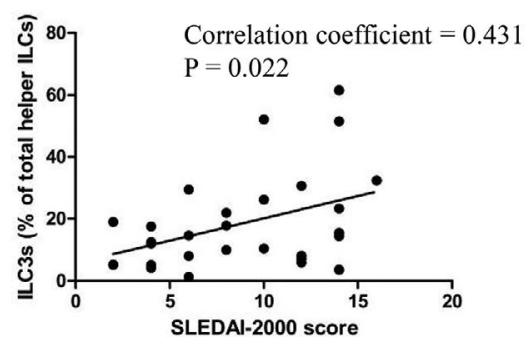

(i)

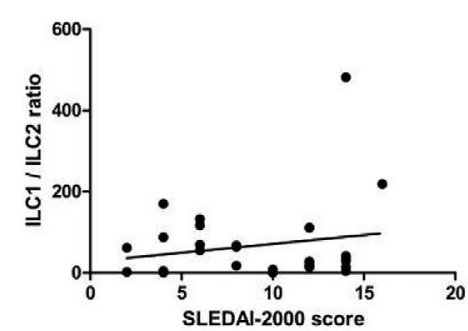

(j)

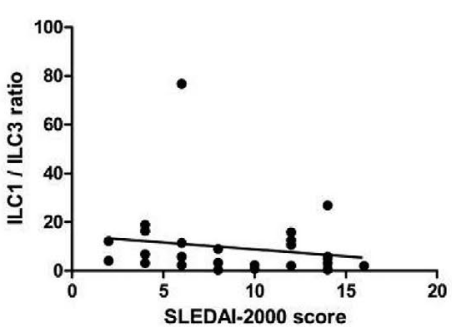

(k)

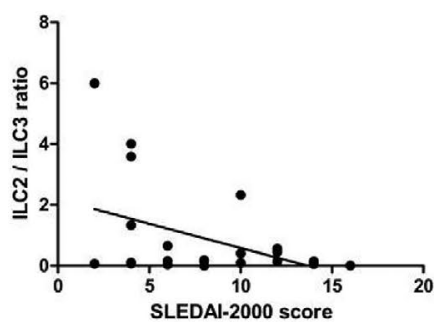

Figure 3. Associations between ILC subsets and ILC ratios and SLEDAI-2000 score. Correlations between total helper ILC numbers (a), total helper ILC frequencies (b), ILC1 numbers (c), ILC1 frequencies (d), ILC2 numbers (e), ILC2 frequencies (f), ILC3 numbers (g), ILC3 frequencies (h), ILC1/ILC2 ratio (i), ILC1/ILC3 ratio (j), and ILC2/ILC3 ratio (k) in patients with systemic lupus erythematosus.

ILC: Innate lymphoid cells; SLEDAI-2000: Systemic Lupus Erythematosus Disease Activity Index 2000; PBMC: Peripheral blood mononuclear cell. Associations were assessed by Spearman correlation coefficient. $\mathrm{P}<0.05$ were considered statistically significant. 
(a)

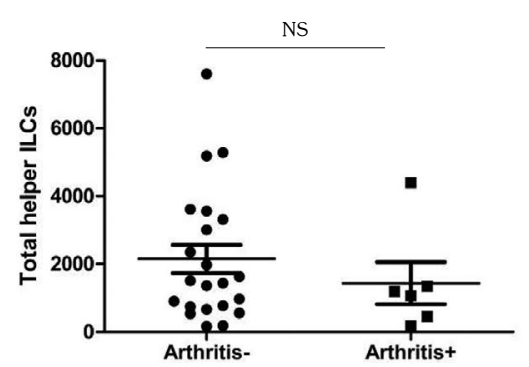

(c)

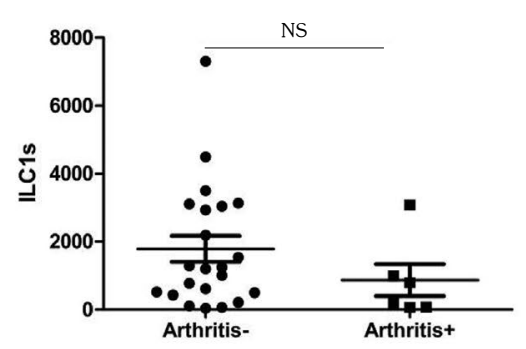

(e)

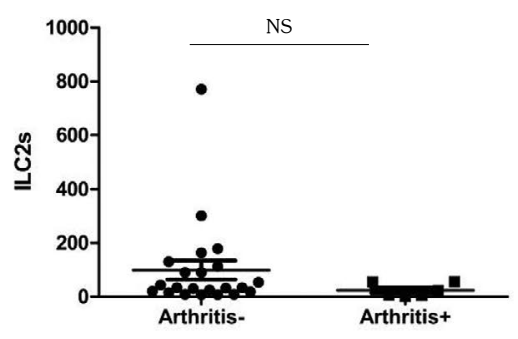

(g)

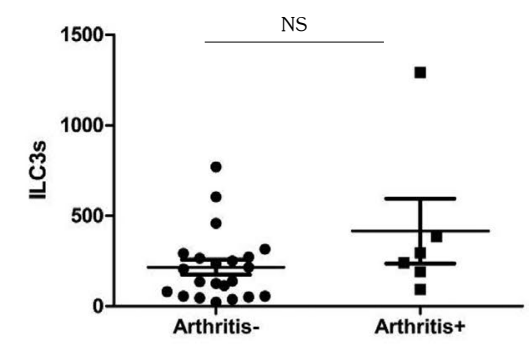

(b)

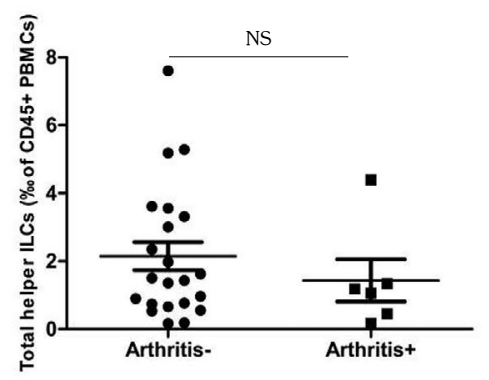

(d)

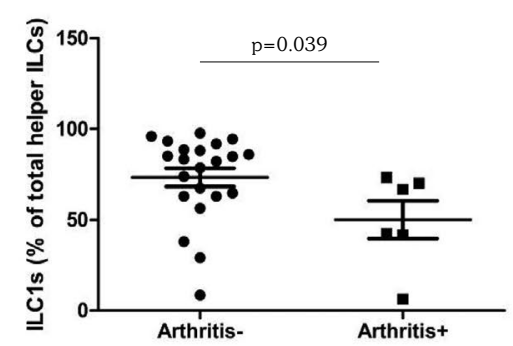

(f)

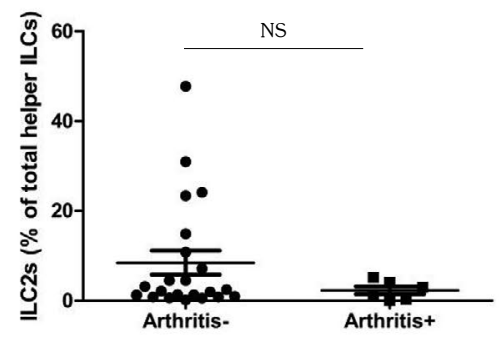

(h)

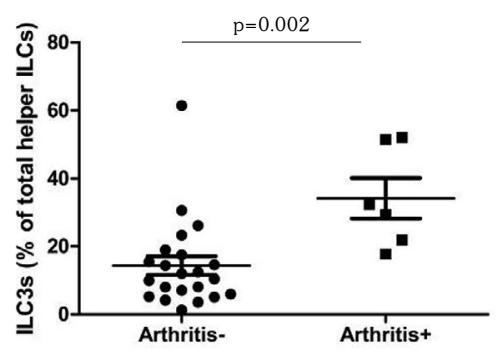

(i)

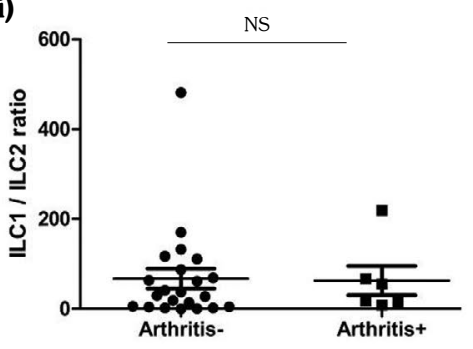

(j)

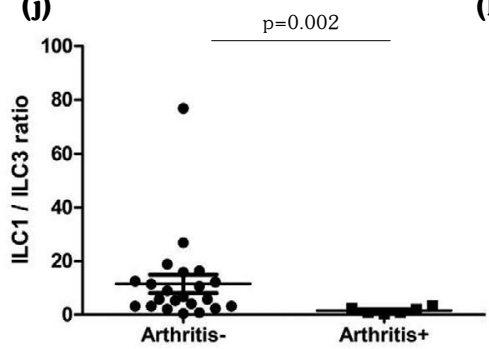

(k)

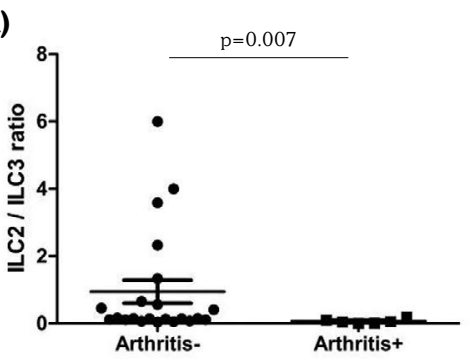

Figure 4. Differences in ILC subsets and ILC ratios between patients with arthritis and patients without arthritis. Comparisons of total helper ILC numbers (a), total helper ILC frequencies (b), ILC1 numbers (c), ILC1 frequencies (d), ILC2 numbers (e), ILC2 frequencies (f), ILC3 numbers (g), ILC3 frequencies (h), ILC1/ILC2 ratio (i), ILC1/ILC3 ratio (j), and ILC2/ILC3 ratio (k) between patients with arthritis $(\mathrm{n}=6)$ and patients without arthritis $(\mathrm{n}=22)$.

ILC: Innate lymphoid cell; NS: Not significant; PBMC: Peripheral blood mononuclear cell. Data are shown as mean \pm standard error of mean. Differences were assessed by Mann-Whitney $\mathrm{U}$ test. $\mathrm{P}<0.05$ were considered statistically significant. 


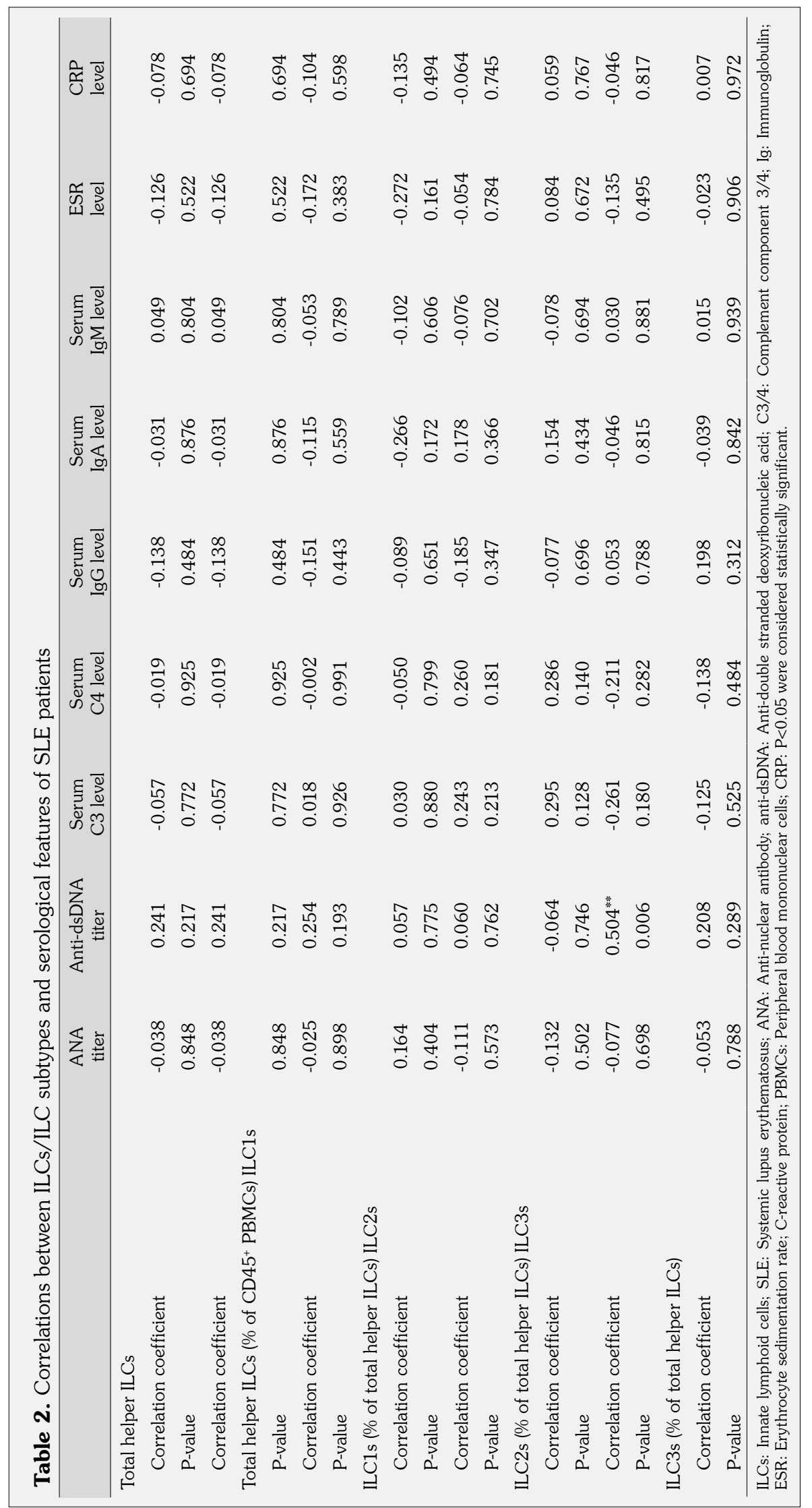


observed. Afterwards, we analyzed the associations between ILC subsets and SLEDAI-2000 score. It was indicated that only the frequencies of ILC3s were positively associated with the SLEDAI-2000 score (correlation coefficient $=0.431, \quad p=0.022$ ) (Figure 3h).

Next, we explored the associations between ILC subsets and organ involvements in patients with SLE. We found that frequencies of ILC1s were lower in SLE patients with arthritis than SLE patients without arthritis $(50.1 \pm 10.4 \%$ and $73.4 \pm 5.0 \%$, respectively; $p=0.039$ ) (Figure $4 d$ ), while frequencies of ILC3s were higher in SLE patients with arthritis than SLE patients without arthritis (34.2 $\pm 6.0 \%$ and $14.3 \pm 2.8$, respectively; $\mathrm{p}=0.002$ ) (Figure $4 \mathrm{~h}$ ). In addition, the ILC1/ILC3 ratio $(1.6 \pm 0.5$ and $11.5 \pm 3.4$, respectively; $\mathrm{p}=0.002$ ) (Figure $4 \mathrm{j}$ ) and the ILC2/ILC3 ratio $(0.1 \pm 0.0$ and $1.0 \pm 0.3$, respectively; $p=0.007)$ (Figure 4k) were decreased in SLE patients with arthritis compared to SLE patients without arthritis. Other ILC subsets did not differ depending on renal system involvement, mucocutaneous system involvement, neurologic system involvement, hematologic system involvement, and lupus with mesenteric vasculitis (data not shown).

Finally, we analyzed the relationships between ILC subsets and the serological features in SLE (including ANA titer, anti-dsDNA titer, serum IgG/A/M level, serum C3/C4 level, ESR level, $\mathrm{CRP}$ level, and other autoantibodies). The results showed that the absolute numbers of ILC3s were positively correlated with serum anti-dsDNA titers (correlation coefficient $=0.504, p=0.006$ ) (Table 2). There were no correlations between other ILC subsets and serological features (data not shown).

\section{DISCUSSION}

Our data revealed an altered distribution of circulating ILC subsets in patients with different disease stages of SLE. In addition, ILC3 frequencies and numbers were positively associated with SLE disease activity. Decreased frequencies of ILC1s and increased frequencies of ILC3s were associated with arthritis involvement in SLE.
Innate lymphoid cells have been recently defined as new players in innate immunity that provide rapid nonspecific responses against foreign antigens. ${ }^{17}$ However, in addition to their protective functions, when dysregulated by the large amounts of cytokines and chemokines that they express, ILCs can have detrimental effects and result in chronic allergic, autoimmune, and inflammatory diseases, including asthma, inflammatory bowel disease, psoriasis, RA, multiple sclerosis (MS), and vasculitis. ${ }^{6,18-22}$ Similar to Th cells, ILC1s mainly produce type 1 cytokines such as IFN- $\gamma$ and tumor necrosis factor; ILC2s mainly produce type 2 cytokines such as interleukin (IL)-4, IL-5, and IL-13; and ILC3s mainly produce type 3 cytokines such as IL-17 and IL-22. ${ }^{23}$

To our knowledge, so far, only two studies have focused on circulating ILCs and distinct ILC subgroups in patients with SLE. According to the findings reported by $\mathrm{Chu}$ et al., ${ }^{12}$ increased frequencies of circulating ILC1s and decreased frequencies of circulating ILC2s and ILC3s were observed. In contrast, Hou and $\mathrm{Liu}^{11}$ identified increased frequencies of ILC1s and ILC 3s, and decreased frequencies of ILC2s in SLE patients compared to those in healthy controls. Unlike the frequencies of ILC2s associated with SLEDAI score and serum IgG level, as well as the ILC1/ILC3 ratio associated with disease activity and nephritis in these two previous studies, we found that ILC3 frequencies were correlated with SLEDAI-2000 score and that the ILC1/ILC3 ratio was related with arthritis. These different findings suggest that SLE is a heterogeneous systemic disease in clinical and cellular phenotypes. Further studies in larger cohorts are warranted to explore how ILCs and ILC subgroups take part in pathogenesis in patients with various SLE clinical manifestations.

In the present study, we identified biased ILC distributions in different SLE disease stages. In patients with active SLE, the ILC1/ILC2 ratio was higher than that in healthy controls; however, in patients with inactive SLE, the ILC1/ILC3 ratio was higher than that in healthy controls. Recent studies have identified that ILC2s and ILC3s could shift to T-bet $^{+}$IFN- $\gamma$-secreting ILC1s depending on IL-12 and IL-18; on the other hand, ILC1s could transdifferentiate back to either ILC2s in response to IL-4 or to ILC3s in response to IL-23 and IL-1 $\beta .{ }^{24}$ Moreover, it has been shown 
that serum IL-12 and IL-18 levels are elevated in SLE patients. ${ }^{25}$ Maazi et al. ${ }^{26}$ also indicated that plasmacytoid dendritic cells (pDCs) suppressed cytokine production and proliferation rates while increasing the rate of apoptosis of ILC2s through IFN- $\alpha$ production in allergic asthma murine models. It is well-known that pDCs are the main source of type I IFN and that the pDC-type I IFN axis plays an important role in the pathogenesis of SLE; moreover, increased pDCs and type I IFN cytokines were found in patients with SLE. ${ }^{27}$ Another report revealed that the proliferation and cytokine production of kidney-resident ILC2s were suppressed by IFN- $\gamma$ and IL-27 produced by activated T cells and myeloid cells in MRL-lpr mice. ${ }^{9}$ However, patients with SLE possess different cytokine profiles depending on the disease activity. ${ }^{28}$ Thus, it may be suggested that various cytokine pathways might contribute to the plasticity of the three ILC subsets in different SLE disease stages.

In our study, we found that the absolute numbers of ILC3s were elevated in patients with active SLE compared with that in patients with inactive SLE, and that ILC3s were positively correlated with SLEDAI-2000 and serum antidsDNA titers. This indicated that ILC3s were associated with disease activity and that it might be a cellular marker to monitor SLE disease flares; however, more prospective studies are needed to confirm this hypothesis.

Systemic lupus erythematosus is a systemic autoimmune disease that can affect almost all organ systems. Our results showed that increased frequencies of ILC3s, decreased frequencies of ILC1s, and decreased ILC1/ILC3 and ILC2/ILC3 ratios were found in patients with arthritis compared to those without arthritis. To our knowledge, to date, there is no research on ILC subsets in patients with SLE accompanied with arthritis. However, many studies on other autoimmune diseases accompanied with arthritis have been reported. Ciccia et al. ${ }^{29}$ found that gutderived ILC3s were expanded in the peripheral blood and synovial fluid in patients with ankylosing spondylitis. Rodríguez-Carrio et al. ${ }^{8}$ found increased lymph node ILC1 and ILC3 counts in patients with RA compared to those in healthy controls. Soare et al. ${ }^{30}$ showed that circulating ILC3 and ILC2 numbers were increased in patients with psoriatic arthritis and that the
ILC2/ILC3 ratio was correlated with disease activity. In addition, one recent study indicated that granulocyte-macrophage colony-stimulating factor-producing ILCs induced by autoimmune Th17 cells could initiate and augment autoimmune arthritis in spontaneous autoimmune arthritis model. ${ }^{31}$ Thus, we suggested that ILC subsets may contribute to the development of autoimmune arthritis and potentially serve as a cellular marker for the diagnosis and monitoring of SLE patients with arthritis. Although we failed to identify significant relationships between ILC subsets and other organ involvements, the link between them needs to be further assessed in the peripheral blood, kidney, skin, and synovial fluid.

A limitation of our study is the small sample size. Thus, larger cohorts will be needed to validate our findings.

In conclusion, the current study compared the distribution of ILC subsets in SLE patients with different clinical disease stages and organ involvements. We found that ILC3 frequencies were correlated with SLE disease activity. In addition, we identified that ILC1 frequencies, ILC3 frequencies, the ILC1/ILC3 ratio, and the ILC2/ILC3 ratio were associated with SLE accompanied with arthritis. Thus, ILC subsets may be cellular markers for SLE disease activity and specifically for SLE accompanied with arthritis.

\section{Declaration of conflicting interests}

The authors declared no conflicts of interest with respect to the authorship and/or publication of this article.

\section{Funding}

The authors received no financial support for the research and/or authorship of this article.

\section{REFERENCES}

1. Tsokos GC. Systemic lupus erythematosus. N Engl J Med 2011;365:2110-21.

2. Liu Z, Davidson A. Taming lupus-a new understanding of pathogenesis is leading to clinical advances. Nat Med 2012;18:871-82.

3. Spits H, Cupedo T. Innate lymphoid cells: emerging insights in development, lineage relationships, and function. Annu Rev Immunol 2012;30:647-75.

4. Artis D, Spits H. The biology of innate lymphoid cells. Nature 2015;517:293-301. 
5. Hazenberg MD, Spits H. Human innate lymphoid cells. Blood 2014;124:700-9.

6. Shikhagaie MM, Germar K, Bal SM, Ros XR, Spits $\mathrm{H}$. Innate lymphoid cells in autoimmunity: emerging regulators in rheumatic diseases. Nat Rev Rheumatol 2017; 13:164-73.

7. Wohlfahrt T, Usherenko S, Englbrecht M, Dees C, Weber S, Beyer C, et al. Type 2 innate lymphoid cell counts are increased in patients with systemic sclerosis and correlate with the extent of fibrosis. Ann Rheum Dis 2016;75:623-6.

8. Rodríguez-Carrio J, Hähnlein JS, Ramwadhdoebe TH, Semmelink JF, Choi IY, van Lienden KP, et al. Brief Report: Altered Innate Lymphoid Cell Subsets in Human Lymph Node Biopsy Specimens Obtained During the At-Risk and Earliest Phases of Rheumatoid Arthritis. Arthritis Rheumatol 2017;69:70-6.

9. Düster M, Becker M, Gnirck AC, Wunderlich M, Panzer U, Turner JE. T cell-derived IFN- $\gamma$ downregulates protective group 2 innate lymphoid cells in murine lupus erythematosus. Eur J Immunol 2018;48:1364-75.

10. Cols M, Rahman A, Maglione PJ, Garcia-Carmona Y, Simchoni N, Ko HM, et al. Expansion of inflammatory innate lymphoid cells in patients with common variable immune deficiency. J Allergy Clin Immunol 2016;137:1206-15.

11. Hou M, Liu S. Innate lymphoid cells are increased in systemic lupus erythematosus. Clin Exp Rheumatol 2019 Feb 15. [Epub ahead of print]

12. Chu H, Fang X, Tan Z, Zhen X, Wu RL, Li XP, et al. Correlation between the changes of innate lymphoid cells in peripheral blood of systemic lupus erythematosus and its clinical significance. Zhonghua Yi Xue Za Zhi 2019;99:169-173.

13. Hochberg MC. Updating the American College of Rheumatology revised criteria for the classification of systemic lupus erythematosus. Arthritis Rheum 1997;40:1725.

14. Gladman DD, Ibañez D, Urowitz MB. Systemic lupus erythematosus disease activity index 2000. J Rheumatol 2002;29:288-91.

15. Björklund ÅK, Forkel M, Picelli S, Konya V, Theorell $\mathrm{J}$, Friberg $\mathrm{D}$, et al. The heterogeneity of human CD127(+) innate lymphoid cells revealed by single-cell RNA sequencing. Nat Immunol 2016;17:451-60.

16. Juelke K, Romagnani C. Differentiation of human innate lymphoid cells (ILCs). Curr Opin Immunol 2016;38:75-85.

17. Spits H, Artis D, Colonna M, Diefenbach A, Di Santo JP, Eberl G, et al. Innate lymphoid cells--a proposal for uniform nomenclature. Nat Rev Immunol 2013;13:145-9.

18. Villanova F, Flutter B, Tosi I, Grys K, Sreeneebus H, Perera GK, et al. Characterization of innate lymphoid cells in human skin and blood demonstrates increase of NKp44+ ILC3 in psoriasis. J Invest Dermatol 2014;134:984-91.
19. Roan F, Stoklasek TA, Whalen E, Molitor JA, Bluestone JA, Buckner JH, et al. CD4+ Group 1 Innate Lymphoid Cells (ILC) Form a Functionally Distinct ILC Subset That Is Increased in Systemic Sclerosis. J Immunol 2016;196:2051-62.

20. Gross CC, Schulte-Mecklenbeck A, Hanning U, Posevitz-Fejfár A, Korsukewitz C, Schwab N, et al. Distinct pattern of lesion distribution in multiple sclerosis is associated with different circulating T-helper and helper-like innate lymphoid cell subsets. Mult Scler 2017;23:1025-30.

21. Degn M, Modvig S, Dyring-Andersen B, Bonefeld $\mathrm{CM}$, Frederiksen JL, Geisler C, et al. Increased prevalence of lymphoid tissue inducer cells in the cerebrospinal fluid of patients with early multiple sclerosis. Mult Scler 2016;22:1013-20.

22. Teunissen MBM, Munneke JM, Bernink JH, Spuls PI, Res PCM, Te Velde A, et al. Composition of innate lymphoid cell subsets in the human skin: enrichment of NCR(+) ILC3 in lesional skin and blood of psoriasis patients. J Invest Dermatol 2014;134:2351-60.

23. Annunziato F, Romagnani C, Romagnani S. The 3 major types of innate and adaptive cellmediated effector immunity. J Allergy Clin Immunol 2015;135:626-35.

24. Serafini N, Vosshenrich CA, Di Santo JP. Transcriptional regulation of innate lymphoid cell fate. Nat Rev Immunol 2015;15:415-28.

25. Wong CK, Ho CY, Li EK, Lam CW. Elevation of proinflammatory cytokine (IL-18, IL-17, IL-12) and Th2 cytokine (IL-4) concentrations in patients with systemic lupus erythematosus. Lupus 2000;9:589-93.

26. Maazi H, Banie H, Aleman Muench GR, Patel N, Wang B, Sankaranarayanan I, et al. Activated plasmacytoid dendritic cells regulate type 2 innate lymphoid cell-mediated airway hyperreactivity. J Allergy Clin Immunol 2018;141:893-905. e6.

27. Panda SK, Kolbeck R, Sanjuan MA. Plasmacytoid dendritic cells in autoimmunity. Curr Opin Immunol 2017;44:20-5.

28. Reynolds JA, McCarthy EM, Haque S, Ngamjanyaporn $\mathrm{P}$, Sergeant JC, Lee E, et al. Cytokine profiling in active and quiescent SLE reveals distinct patient subpopulations. Arthritis Res Ther 2018;20:173

29. Ciccia F, Guggino G, Rizzo A, Saieva L, Peralta S, Giardina A, et al. Type 3 innate lymphoid cells producing IL-17 and IL-22 are expanded in the gut, in the peripheral blood, synovial fluid and bone marrow of patients with ankylosing spondylitis. Ann Rheum Dis 2015;74:1739-47.

30. Soare A, Weber S, Maul L, Rauber S, Gheorghiu AM, Luber $\mathrm{M}$, et al. Cutting edge: homeostasis of innate lymphoid cells is imbalanced in psoriatic arthritis. $\mathrm{J}$ Immunol 2018;200:1249-54.

31. Hirota K, Hashimoto M, Ito Y, Matsuura M, Ito H, Tanaka M, et al. Autoimmune Th17 cells induced synovial stromal and innate lymphoid cell secretion of the cytokine GM-CSF to initiate and augment autoimmune arthritis. Immunity 2018;48:1220-32. 